\title{
CARACTERIZAÇÃO DE IDOSOS VÍTIMAS DE ACIDENTES POR CAUSAS EXTERNAS
}

Clóris Regina Blanski Grden', Jacy Aurelia Vieira de Sousa1, Maria Helena Lenardt², Regianne Mara Pesck³, Márcia Daniele Seima ${ }^{4}$, Pollyanna Kássia de Oliveira Borges ${ }^{5}$

${ }^{1}$ Enfermeira. Doutoranda em Enfermagem. Universidade Federal do Paraná. Curitiba-PR-Brasil.

${ }^{2}$ Enfermeira. Doutora em Filosofia. Universidade Federal do Paraná. Curitiba-PR-Brasil.

${ }^{3}$ Enfermeira. Prefeitura Municipal de Imbituva. Imbituva-PR-Brasil.

${ }^{4}$ Enfermeira. Doutora em Enfermagem. Universidade Federal do Paraná. Curitiba-PR-Brasil.

${ }^{5}$ Dentista. Doutora em Saúde Coletiva. Universidade Estadual de Ponta Grossa. Ponta Grossa-PR-Brasil.

RESUMO: Estudo quantitativo retrospectivo, cujo objetivo foi caracterizar as ocorrências em idosos vítimas de acidentes por causas externas, atendidos por um Serviço de Atendimento Móvel de Urgência, em uma cidade do Estado do Paraná, Brasil. A amostra compreendeu 324 ocorrências no período amostral de junho a dezembro de 2009. Foram respeitados os preceitos éticos no estudo. A maioria das vítimas era do sexo feminino ( $n=179 ; 55,25 \%)$, na faixa etária de 60 a 65 anos, com Hipertensão Arterial Sistêmica $(n=80 ; 24,69 \%)$ e Diabetes Mellitus ( $n=33 ; 10,18 \%$ ). Foram significativas as ocorrências nos domicílios ( $n=171 ; 52,78 \%)$; a maior incidência foi das quedas de mesmo nível ( $n=185 ; 57,10 \%)$, seguidas dos acidentes de transporte terrestre $(n=73 ; 22,52 \%)$. Destaca-se a importância de ações pela equipe multiprofissional de saúde, voltadas à prevenção de acidentes por causas externas na população idosa, com ênfase em informações sobre os riscos e cuidados preventivos nas quedas de mesmo nível em domicílio.

DESCRITORES: Idoso; Causas externas; Acidentes por quedas.

\section{CHARACTERIZATION OF ELDERLY ACCIDENT VICTIMS DUE TO EXTERNAL CAUSES}

\begin{abstract}
The objective in this quantitative and retrospective study was to characterize accident events in elderly victims due to external causes, attended by a Mobile Emergency Care Service in a city in the State of Paraná, Brazil. The sample consisted of 324 events, during the sample period from June to December 2009. The ethical premises were respected in the study. Most of the victims were female $(\mathrm{N}=179 ; 55.25 \%)$, in the age range from 60 to 65 years, suffering from Systemic Arterial Hypertension ( $\mathrm{N}=80 ; 24.69 \%)$ and Diabetes Mellitus ( $\mathrm{N}=33 ; 10.18 \%)$. Events at home were significant $(\mathrm{N}=171 ; 52.78 \%)$; the highest incidence level was related to falls from the same height $(\mathrm{N}=185 ; 57.10 \%)$, followed by ground transportation accidents $(\mathrm{N}=73 ; 22.52 \%)$. The importance of actions by the multiprofessional health team is highlighted, focused on the prevention of accidents due to external causes in the elderly population, emphasizing information about risks and preventive care in cases of falls from the same height at home.
\end{abstract}

DESCRIPTORS: Aged; External causes; Accidental falls.

\section{CARACTERIZACIÓN DE ANCIANOS VÍCTIMAS DE ACCIDENTES POR CAUSAS EXTERNAS}

RESUMEN: Estudio cuantitativo retrospectivo, cuyo objetivo fue caracterizar las ocurrencias en ancianos víctimas de accidentes por causas externas, atendidos por un Servicio de Atendimiento Movil de Urgencia, en una ciudad del Estado de Paraná, Brasil. La muestra abarcó 324 ocurrencias en el periodo de junio a diciembre de 2009. Fueron respetados los preceptos éticos en el estudio. La mayoría de las víctimas era del sexo femenino $(\mathrm{n}=179 ; 55,25 \%)$, en la franja etaria de 60 a 65 años, con Hipertensión Arterial Sistémica $(n=80 ; 24,69 \%)$ y Diabetes Mellitus ( $n=33 ; 10,18 \%)$. Fueron significativas las ocurrencias en los domicilios $(n=171 ; 52,78 \%)$; la mayor incidencia fue de las caídas de mismo nivel $(n=185$; $57,10 \%)$, seguidas de los accidentes de transporte terrestre $(\mathrm{n}=73 ; 22,52 \%)$. Se destaca la importancia de acciones por el equipo multiprofesional de salud, para la prevención de accidentes por causas externas en la población anciana, con énfasis en informaciones sobre los riesgos y cuidados preventivos en las caídas de mismo nivel en domicilio. DESCRIPTORES: Anciano; Causas externas; Accidentes por caídas.
Autor Correspondente:

Clóris Regina Blanski Grden

Universidade Federal do Paraná

Rua Teodoro Sampaio, 888 - 84036-070 - Ponta Grossa-PR-Brasil

E-mail: reginablanski@hotmail.com
Recebido: 18/06/2013

Finalizado: $24 / 06 / 2014$ 


\section{INTRODUÇÃO}

Os acidentes por causas externas estão entre as principais ocorrências de morte na população geral e entre os idosos. Segundo os dados disponíveis no Departamento de Informática do Sistema Único de Saúde, no ano de 2011, as causas externas foram a terceira causa de óbito na população geral ( $n=145.842$ óbitos) e a sétima causa de morte entre os indivíduos com idade acima de 60 anos $\left(n=24.669\right.$ óbitos) ${ }^{(1)}$. Compreende-se por causas externas as lesões, sejam físicas, mentais ou psicológicas, que podem ou não levar ao óbito, decorrentes de acidentes (trânsito, afogamento, envenenamento, quedas, queimaduras) e violência (agressão/ homicídio, suicídio, abuso físico, sexual e psicológico) $)^{(2-4)}$.

Os acidentes e a violência configuramse como as principais causas de morte na população jovem e adulta, no entanto, com o avançar da idade as taxas de mortalidade nos idosos tem se apresentado crescentes. Em 2013, a mortalidade por causas externas na população paranaense, independente da idade, foi de 83,7 óbitos/100.000 habitantes. Especificamente para os idosos, no Paraná e na região Sul, respectivamente 1679 e 3825 morreram por causas externas neste mesmo ano ${ }^{(1)}$.

Desta forma, a ocorrência de agravos, caracterizados por acidentes e violências, configura-se um sério problema de saúde pública no mundo, com forte impacto na morbimortalidade da população(5) e a identificação do perfil das ocorrências, bem como a caracterização sociodemográfica e clínica das vítimas, possibilita o direcionamento de políticas públicas voltadas à minimização desses eventos que podem ser evitáveis. Igualmente, os atendimentos que se caracterizam como quadros agudos de urgência devem ser objeto de preocupação entre os profissionais da área da saúde, pois lesões que poderiam ser facilmente toleradas por pacientes jovens podem resultar em índices consideráveis de mortalidade no idoso ${ }^{(6)}$.

Nos serviços de emergência, as quedas se apresentam como um dos principais motivos de atendimento. Segundo o relatório global da Organização Mundial da Saúde (OMS), esse evento responde por $40 \%$ das mortes relacionadas a ferimentos, ou seja, aproximadamente $28 \%$ a
35\% das pessoas com mais de 65 anos sofrem quedas a cada ano, sendo que a frequência aumenta com a idade e o nível de fragilidade do idoso ${ }^{(7)}$. Estudos apontam que as quedas configuram-se como causa de morte em idosos, seguida pelos Acidentes de Transporte Terrestre, afogamentos e queimaduras, causas externas indeterminadas, agressões e suicídios ${ }^{(2,4)}$. Além disso, demonstram que esses eventos ocorrem predominantemente no domicílio e com pouca contribuição do consumo de álcool| ${ }^{(8-9)}$.

Justifica-se a realização do estudo pelo fato que os eventos, anteriormente descritos, podem gerar sequelas no idoso como a redução da capacidade funcional, limitação das atividades básicas de vida, dependência, depressão, diminuição da qualidade de vida, e até mesmo, a morte ${ }^{(10-11)}$. Além disso, no Brasil, o número de hospitalizações de idosos devido a essas causas mostra-se relevante, com 413.139 internações, entre 2008 e 2010, com custo aproximado de 570 milhões de reais ${ }^{(12)}$.

O presente estudo tem como objetivo caracterizar as ocorrências em idosos vítimas de acidentes por causas externas, atendidos por um Serviço de Atendimento Móvel de Urgência, quanto ao local e natureza, bem como as lesões e sinais sintomas.

\section{MÉTODO}

Trata-se de estudo quantitativo retrospectivo, no qual foram utilizados dados secundários contidos nas fichas de ocorrência de um Serviço de Atendimento Móvel de Urgência, de uma cidade do Paraná-Brasil. A circunscrição territorial do município deste estudo está localizada na região dos Campos Gerais, numa área de 2.025,697 km2. Em 2009, com uma população total estimada de 314.527 habitantes, 32.320 habitantes tinham idade igual ou superior a 60 $\operatorname{anos}^{(13)}$.

Atualmente, o sistema de saúde do município apresenta uma estrutura com 51 Unidades Básicas de Saúde, além de 39 equipes de saúde da família, quatro centros de atenção à saúde, uma Unidade de Atenção Básica para os idosos e um hospital municipal geral. A rede de urgência e emergência está em fase de implantação no município e no caso de um evento dessa natureza, o idoso segue 
a linha de cuidado aplicada a todas as faixas etárias neste sistema.

A coleta de dados foi realizada de abril a julho de 2010. A amostra foi constituída pelo total de ocorrências com idosos, ou seja, 324 vítimas de causas externas (acidentes e/ou violência), atendidos no período amostral de junho a dezembro de 2009. Os dados foram coletados por meio de instrumento adaptado da ficha de atendimento do serviço, na qual as variáveis selecionadas foram: sexo, idade, morbidades da vítima, local e natureza da ocorrência, lesões, sinais e sintomas apresentados no momento da ocorrência. Após a coleta, os dados foram armazenados no programa Microsoft Office Excel $2007^{\circledR}$ e posteriormente submetidos à estatística descritiva, com demonstração dos resultados por meio de tabelas com frequência absoluta e percentual.

O trabalho foi encaminhado ao Comitê de Ética e Pesquisa da Universidade Estadual de Ponta Grossa e recebeu parecer favorável sob o n. 18/2010, na data de 30 de abril de 2010.

\section{RESULTADOS}

Aponta-se na Tabela 1 que de 324 atendimentos aos idosos, vítimas de acidentes por causas externas, 55,25\% $(n=179)$ dos idosos eram do sexo feminino, na faixa etária de 60 a 65 anos $(n=85 ; 26,24 \%)$, seguida de 80 anos ou mais ( $\mathrm{n}=80 ; 24,69 \%)$. As morbidades foram a Hipertensão Arterial Sistêmica $(n=80 ; 24,69 \%)$ e o Diabetes Mellitus ( $\mathrm{n}=33 ; 10,18 \%$ ).

Quanto ao local da ocorrência predominou o domicílio ( $\mathrm{n}=171 ; 52,78 \%$ ), e em via pública $(n=135 ; 41,67 \%)$. Foram mais frequentes as quedas de mesmo nível ( $n=185 ; 57,10 \%)$; seguidas por acidentes de transporte terrestre $(n=73 ; 22,52 \%)$. As quedas de planos elevados compuseram 10,8\% $(n=35)$ das ocorrências atendidas (Tabela 2).

Visualiza-se no Quadro 1 que as principais lesões sofridas foram as contusões $(n=158)$, escoriações $(n=102)$, os ferimentos contusos $(n=95)$ e as fraturas $(n=63)$. As regiões corpóreas mais atingidas foram os membros inferiores $(n=127)$, membros superiores $(n=107)$ e crânio $(\mathrm{n}=105)$.

Destaca-se a dor como o principal sintoma apresentado pelos idosos ( $\mathrm{n}=159 ; 49,07 \%)$; e todos eles apresentaram mais de um sinal ou sintoma durante o atendimento (Tabela 3). No entanto, os dados referentes a sinais e sintomas não estavam preenchidos na sua totalidade nas fichas de ocorrência.

Referente aos encaminhamentos das vítimas tem-se o hospital público municipal com $n=229$ (70,68\%) idosos, visto que é a instituição de referência para o atendimento no município e apenas n=95 (29,32\%) foram encaminhados para outros serviços.

Tabela 1 - Distribuição de idosos vítimas de acidentes por causas externas atendidos por um serviço móvel de urgência, segundo sexo, idade e morbidades. Ponta Grossa-PR-Brasil, 2010

\begin{tabular}{lcc}
\hline Sexo & $\mathbf{n}$ & $\mathbf{\%}$ \\
\hline Feminino & 179 & $55,25 \%$ \\
\hline Masculino & 145 & $44,75 \%$ \\
\hline Idade & $\mathbf{n}$ & $\mathbf{\%}$ \\
\hline 60 -| 65 anos & 85 & $26,24 \%$ \\
\hline 65 -| 70 anos & 50 & $15,43 \%$ \\
\hline 70 -| 75 anos & 65 & $20,06 \%$ \\
\hline 75 -| 80 anos & 44 & $13,58 \%$ \\
\hline$>80$ anos & 80 & $24,69 \%$ \\
\hline Morbidades & $\mathbf{n}$ & $\mathbf{\%}$ \\
\hline Hipertensão Arterial & 80 & $24,69 \%$ \\
\hline Sistêmica & 33 & $10,18 \%$ \\
\hline Diabetes Mellitus & 16 & $4,94 \%$ \\
\hline Doença Cardiovascular & 08 & $2,47 \%$ \\
\hline Acidente Vascular & 06 & $1,85 \%$ \\
\hline Cerebral & 05 & $1,54 \%$ \\
\hline Doença Respiratória & 04 & $1,23 \%$ \\
\hline Convulsão & 03 & $0,92 \%$ \\
\hline Alcoolismo & 02 & $0,62 \%$ \\
\hline Doença Mental & 01 & $0,30 \%$ \\
\hline Doença Renal & 49 & $15,12 \%$ \\
\hline Aids & 123 & $37,92 \%$ \\
\hline Outros & & \\
\hline Não Informado & & \\
\hline
\end{tabular}

Nota: A totalidade dos antecedentes clínicos ultrapassou $100 \%$ devido à ocorrência de mais de um antecedente por indivíduo. 
Tabela 2 - Distribuição do local e natureza de ocorrências, com idosos vítimas de acidentes por causas externas atendidos por um serviço de atendimento móvel de urgência. Ponta Grossa-PR-Brasil, 2010

\begin{tabular}{lcc}
\hline Local da ocorrência & $\mathbf{n}$ & $\mathbf{\%}$ \\
\hline Domicílio & 171 & $52,78 \%$ \\
\hline Via Pública & 135 & $41,67 \%$ \\
\hline Estabelecimento Comercial & 05 & $1,54 \%$ \\
\hline Local de Trabalho & 01 & $0,31 \%$ \\
\hline Outros & 12 & $3,70 \%$ \\
\hline Natureza da ocorrência & $\mathbf{n}$ & $\mathbf{\%}$ \\
\hline Queda de Mesmo Nível & 185 & $57,10 \%$ \\
\hline Acidente de Transporte Terrestre & 73 & $22,52 \%$ \\
\hline Queda de Plano Elevado & 35 & $10,8 \%$ \\
\hline Violência & 10 & $3,10 \%$ \\
\hline Acidente com Animal & 04 & $1,23 \%$ \\
\hline Queimadura & 03 & $0,93 \%$ \\
\hline Ferimento por Arma Branca & 02 & $0,62 \%$ \\
\hline Outros & 12 & $3,7 \%$ \\
\hline
\end{tabular}

Quadro 1 - Distribuição de lesões identificadas em região corpórea de idosos, vítimas de acidentes por causas externas atendidos por um serviço de atendimento móvel de urgência. Ponta Grossa-PR-Brasil, 2010

\begin{tabular}{|l|l|l|l|l|l|l|l|l|}
\hline $\begin{array}{l}\text { Tipos de } \\
\text { lesões }\end{array}$ & Crânio & $\begin{array}{l}\text { Face/ } \\
\text { Pescoço }\end{array}$ & Dorso & $\begin{array}{l}\text { Tórax/ } \\
\text { Abdômem }\end{array}$ & Pelve & MMSS* $^{*}$ & MMI** $^{*}$ Total \\
\hline Contusão & 32 & 23 & 11 & 22 & 12 & 22 & 36 & 158 \\
\hline $\begin{array}{l}\text { Esco- } \\
\text { riações }\end{array}$ & 17 & 12 & 03 & 03 & - & 43 & 24 & 102 \\
\hline $\begin{array}{l}\text { Ferimen- } \\
\text { to contu- } \\
\text { so }\end{array}$ & 52 & 28 & - & - & - & 06 & 09 & 95 \\
\hline Fratura & - & 05 & - & 05 & 01 & 13 & 39 & 63 \\
\hline Luxação & 01 & - & - & - & 02 & 08 & 10 & 21 \\
\hline $\begin{array}{l}\text { Lacer- } \\
\text { ação }\end{array}$ & 03 & 04 & - & - & - & 10 & 01 & 18 \\
\hline $\begin{array}{l}\text { Fratura } \\
\text { Exposta }\end{array}$ & - & - & - & - & - & 02 & 06 & 08 \\
\hline $\begin{array}{l}\text { Ferimen- } \\
\text { to Perfu- } \\
\text { rante }\end{array}$ & - & 01 & - & 02 & - & 01 & 01 & 05 \\
\hline $\begin{array}{l}\text { Quei- } \\
\text { madura }\end{array}$ & - & 03 & - & - & - & 01 & 01 & 05 \\
\hline $\begin{array}{l}\text { Am- } \\
\text { putação }\end{array}$ & - & - & - & - & - & 01 & - & 01 \\
\hline Total & 105 & 76 & 14 & 32 & 15 & 107 & 127 & 476 \\
\hline
\end{tabular}

Nota: O total ultrapassou a amostra devido à ocorrência de mais de uma lesão por individuo e em várias regiões do corpo.

*Membros superiores; ${ }^{* *}$ Membros inferiores. 
Tabela 3 - Distribuição de sinais e sintomas apresentados por idosos, vítimas de acidentes por causas externas atendidos por um Serviço de Atendimento Móvel de Urgência. Ponta Grossa-PR-Brasil, 2010

\begin{tabular}{lcc}
\hline Sinais e sintomas & $\mathbf{n}$ & $\mathbf{\%}$ \\
\hline Dor & 159 & 49,07 \\
\hline Sangramento & 19 & 5,86 \\
\hline Palidez & 8 & 2,47 \\
\hline Vômito/Náuseas & 8 & 2,47 \\
\hline Agitação & 7 & 2,16 \\
\hline Inconsciência & 5 & 1,54 \\
\hline Convulsão & 4 & 1,23 \\
\hline Ausência de Pulso & 1 & 0,31 \\
\hline Cianose & 1 & 0,31 \\
\hline Outros & 50 & 15,43 \\
\hline
\end{tabular}

\section{DISCUSSÃO}

Os acidentes de causas externas revelam predominância do sexo feminino e, dos 324 atendimentos, mais da metade decorrem de eventos no próprio domicílio (52,78\%), o que pode ser justificado pelo exercício de atividades domésticas das mulheres (aposentadas ou donas de casa) nessa fase da vida. Outros fatores a serem considerados são as barreiras arquitetônicas do ambiente e a polifarmácia predominantemente no sexo feminino, em especial os psicotrópicos, que podem aumentar o risco para quedas ${ }^{(14)}$. Estudos brasileiros ${ }^{(2,9)}$ e internacionais ${ }^{(15-16)}$ sobre o tema encontraram resultados semelhantes aos apresentados.

A incidência dos eventos foi maior na faixa etária dos 60 a 65 anos. Pesquisas ${ }^{(17-19)}$ afirmam que a maioria desse segmento etário apresentam independência e autonomia, e ainda atividades de trabalho, lazer e social. São características de uma população adulta que os torna mais exposta aos riscos de acidente por causas externas.

Foi significativo também o número de ocorrências nos idosos acima 80 anos. Em estudo $^{(19)}$ realizado em Londrina-PR, Brasil, com 108 idosos vítimas de trauma, assistidos em unidade de urgência e emergência, 33\% compreendiam esta faixa etária. Compreendese que com o aumento da idade, alterações anatômicas e fisiológicas decorrentes do processo de envelhecimento, podem comprometer o desempenho funcional do idoso e tornando-o mais suscetível aos acidentes por causas externas.

Observa-se a ocorrência da Hipertensão
Arterial Sistêmica (HAS) e do Diabetes Mellitus (DM), situação esperada visto que, são doenças consideradas de incidência significativa em idosos. No Brasil, a frequência do diagnóstico médico prévio de HAS e DM atingiram, respectivamente, $59,7 \%$ e 21,6\% em indivíduos com 65 anos ou mais no ano de $2009^{(10)}$. Esse achado se assemelha a outros estudos em que foi encontrada elevada incidência de comorbidades entre os idosos vítimas de causas externas ${ }^{(6,9,19)}$.

A presença de doenças crônicas e comorbidades, associadas a um perfil dinâmico do idoso contemporâneo, oportuniza a ocorrência das quedas, resultando em significativas consequências psicológicas, físicas e sociais para a vida desses indivíduos.

Referente à natureza do chamado, as quedas do mesmo nível representaram mais da metade das ocorrências (57,10\%). A literatura aponta dados semelhantes em estudos nacionais ${ }^{(9,11)}$ e atribui aos acidentes causas como: perda da consciência, escorregões ou tropeços em superfícies lisas ou molhadas. Destaca-se que são acidentes que poderiam ser amenizados ou evitados por meio de programas e medidas de prevenção de quedas.

As quedas são consideradas um fenômeno complexo, influenciadas por diversos aspectos intrínsecos e extrínsecos ao indivíduo e que resultam, na maioria dos eventos, em lesões diversas $^{(4,20)}$, podem causar escoriações, fraturas diversas, traumatismo craniano até o óbito do idoso, e são evidenciadas como responsáveis pela maior proporção de mortes, hospitalizações e atendimentos de emergências ${ }^{(7)}$. 
Na área da Gerontologia são fundamentais as estratégias de cuidados profissionais para a prevenção de quedas em idosos. São orientações de cuidados que se efetivam por meio de ações de conscientização dos idosos e familiares, as quais envolvem informações sobre os riscos e os cuidados, e esses exigem mudanças comportamentais (estilo de vida) e modificações ambientais (barreiras arquitetônicas). Isso somente se viabiliza mediante capacitação dos profissionais de saúde referente à prevenção e o gerenciamento de quedas.

O modelo teórico de prevenção de quedas da $\mathrm{OMS}^{(2)}$ pode ser utilizado por se tratar de uma estratégia articulada, sistemática e abrangente; esse tem como objetivo conscientizar sobre a importância da prevenção e do tratamento das lesões decorrentes das quedas, bem como melhorar a avaliação dos diversos fatores causais e a implementação de intervenções que possam reduzir a ocorrência nos idosos.

O número de ocorrências por ATT foi expressivo, estudos internacionais apontam o significativo aumento desta ocorrência, bem como os elevados índices de mortalidade nos indivíduos acima dos 60 anos $^{(21-23)}$. Em geral, este segmento etário se envolvem com mais frequência em atropelamentos e com multiplicidade de lesões que ocasionam o óbito ${ }^{(19)}$.

As quedas e os acidentes de transporte terrestre podem ser mais frequentes nos idosos em virtude das alterações fisiológicas provenientes do próprio processo de envelhecimento, como redução da velocidade da marcha, déficit da acuidade visual e auditiva, alterações físicas e cognitivas provenientes de doenças e inadequação de ambientes domiciliares nas quais residem os idosos.

Destaca-se que o número de ocorrências decorrentes de violência a idosos não foram significativos, o que pode ser justificado pela frequente subnotificação do evento aos serviços de saúde. Dessa maneira, complicações provenientes de acidentes e violência, como o óbito, tendem a ser caracterizadas como uma complicação oriunda do trauma ${ }^{(24-25)}$. Para a OMS a violência contra os mais velhos apesar de não ser um fenômeno recente, possui extensão ainda pouco conhecida ${ }^{(7)}$.
Quanto à distribuição de lesões identificadas no idoso, observa-se a predominância de contusões, escoriações, ferimentos contusos e fraturas. Estudo realizado na cidade de Campinas, apontou que os traumatismos cranianos e as lesões na face são ocorrências comumente encontradas no idoso traumatizado ${ }^{(6)}$.

As lesões específicas do tipo fraturas foram encontradas em apenas 19,44\% dos idosos, fato que diverge de estudos internacionais, que indicam que essa tem sido uma das consequências mais significativas em relação a causas externas, especialmente às quedas ${ }^{(16,26)}$. Ressalta-se que as contusões e principalmente as fraturas, podem causar imobilidade no idoso, interferindo na realização das atividades básicas de vida diária, podendo levá-lo a uma situação de dependência e perda da autonomia.

Referente aos sinais e sintomas registrados, destaca-se a dor dentre outras manifestações clínicas. Esse dado corrobora aos resultados do estudo desenvolvido em um setor de emergência, em que cerca de $80 \%$ dos pacientes admitidos referiam dor, especialmente após eventos de trauma ${ }^{(19)}$.

Destaca-se que as limitações da presente investigação decorrem do seu caráter documental que pode comprometer a realidade do fenômeno pesquisado, posto que variável morbidade pode ter sido subnotificada. Além disso, a qualidade das informações, como o uso de letra ilegível na ficha de atendimento; dados incompletos, dentre outros, dificultou a coleta das informações para o estudo.

\section{CONCLUSÃO}

As ocorrências em idosos vítimas de acidentes por causas externas apontou o predomínio de mulheres, na faixa etária entre 60 a 65 anos, hipertensas, que sofreram acidente em seus domicílios, principalmente em quedas do mesmo nível, com lesões do tipo contusões e escoriações.

Além das consequências físicas, em geral, os efeitos psicológicos e sociais provenientes de acidentes por causas externas não são considerados no atendimento a ocorrências de urgência/emergência. Salienta-se que, no paciente idoso, esses desfechos mostram-se relevantes e podem gerar consequências a curto 
e longo prazo, podendo evoluir para o óbito.

Conclui-se que a caracterização de idosos vítimas de acidentes por causas externas colabora para a reflexão acerca desse fenômeno, que representa um problema de saúde pública, favorecendo a elaboração de educativas e de prevenção direcionadas a esse grupo etário.

\section{REFERÊNCIAS}

1. Ministério da Saúde (BR). Informações de Saúde. Departamento de Informática do SUS- DATASUS. [Internet] [acesso em 12 mai 2014]. Disponível: http:// www.datasus.gov.br

2. Mascarenhas MDM, Monteiro RA, Sá NNB, Gonzaga LAA, Neves ACM, Roza DL, et al. Epidemiologia das causas externas no Brasil: mortalidade por acidentes e violências no período de 2000 a 2009. In: Ministério da Saúde (BR). Saúde Brasil 2010: Uma análise da situação de saúde e de evidências selecionadas de impacto de ações de vigilância em saúde. Brasília: Ministério da Saúde; 2011. p. 225-49.

3. Ministério da Saúde (BR). Portaria n. 737, de 16 de maio de 2001. Dispõe sobre a política nacional de redução da morbimortalidade por acidentes e violências. Diário Oficial da União. Brasília; 2001.

4. World Health Organization. Injury and Violence: the Facts. Geneva; 2010. [Internet] [acesso em 25 set 2012]. Disponível: http://whqlibdoc.who.int/ publications/2010/9789241599375_eng.pdf?ua=1

5. Matos KF, Martins CB. Mortalidade por causas externas em crianças, adolescentes e jovens: uma revisão bibliográfica. Rev. espaço saúde. [Internet] 2013;14(1) [acesso em 2 set 2013]. Disponível: http://www.uel. br/revistas/uel/index.php/espacoparasaude/article/ view/10480

6. Lima RS, Campos MLP. Perfil do idoso vítima de trauma atendido em uma unidade de urgência e emergência. Rev Esc Enferm USP. [Internet] 2011;45(3) [acesso em 02 ago 2012]. Disponível: http://dx.doi. org/10.1590/S0080-62342011000300016

7. World Health Organization. Relatório global da OMS sobre prevenção de quedas na velhice. Geneva: World Health Organizatio; 2002. 64 p.

8. Luz TCB, Malta DC, Sá NNB, Silva MMA, LimaCosta MF. Violências e acidentes entre adultos mais velhos em comparação aos mais jovens: evidências do sistema de vigilância de violências e acidentes (VIVA), Brasil. Cad. Saúde Pública. [Internet] 2011; 27(11) [acesso em 20 ago 2012]. Disponível: http:// dx.doi.org/10.1590/S0102-311X2011001100007
9. Gomes LMX, Barbosa TLA, Caldeira AP. Mortalidade por causas externas em Minas Gerais, Brasil. Esc Anna Nery. [Internet] 2010;14(4). [acesso em 25 set 2012]. Disponível: http://dx.doi.org/10.1590/S141381232013000800017

10. Mesquita GV, Lima MALTA, Santos AMR, Alves ELM, Brito JNPO, Martins MCC. Morbimortalidade em idosos por fratura proximal do fêmur. Texto Contexto Enferm. [Internet] 2009;18(1). [acesso em 2 set 2012]. Disponível: http://dx.doi.org/10.1590/S010407072009000100008

11. Ribeiro AP, Souza ER, Atie S, Souza AC, Schilithz AO. A influência das quedas na qualidade de vida de idosos. Ciênc. saúde colet. [Internet] 2008;13(4). [acesso em 5 set 2012]. Disponível: http://dx.doi.org/10.1590/S141381232008000400023

12. Ministério da Saúde (BR). Informações de saúde. Departamento de Informática do SUS-DATASUS. [Internet] [acesso em 15 set 2012]. Disponível: http:// www2.datasus.gov.br/DATASUS/index.php?area=02.

13. Instituto Brasileiro de Geografia e Estatística (IBJE). Censo 2010. [Internet] [acesso em 22 set 2012]. Disponível: http://www.ibge.gov.br/home/estatistica/ populacao/censo2010/default.shtm

14. Olazarán J, Valle D, Serra JA, Cano P, Muñiz R. Psychotropic medications and falls in nursing homes: a cross-sectional study. J Am Med Dir Assoc. 2013;14(3):213-7.

15. Hsia RY, Wang E, Saynina O, Wise P, Pérez-Stable EJ, Auerbach A. Factors associated with trauma center use for elderly patients with trauma: a statewide analysis, 1999-2008. Arch Surg. [Internet] 2011;146(5) [acesso em 10 ago 2012]. Disponível: http://www.ncbi.nlm. nih.gov/pmc/articles/PMC3121677/

16. Alexandrescu R, O'Brien SJ, Lecky FE. A review of injury epidemiology in the UK and Europe: some methodological considerations in constructing rates. BMC Public Health. [Internet] 2009;9(226). [acesso em 10 ago 2012]. Disponível: http://www.biomedcentral. com/1471-2458/9/226

17. Zakariassen E, Burman RA, Hunskaar S. The epidemiology of medical emergency contacts outside hospitals in Norway - a prospective population based study. Scandinavian J. Trauma, Resuscitation Emerg. Med. [Internet] 2010;18(9). [acesso em 18 ago 2012]. Disponível: http://www. sjtrem.com/content/18/1/9

18. Mathias TAF, Jorge MHP, Andrade OG. Mortalidade por causas externas na população idosa residente em município da região sul do Brasil. Rev. Latino-Am. Enfermagem. [Internet] 2006;14(1). [acesso em 10 ago 2012]. Disponível: http://dx.doi.org/10.1590/S141481452010000400018 
19. Biazin DT, Rodrigues RAP. Perfil dos idosos que sofreram trauma em Londrina - Paraná. Rev Esc Enferm USP. [Internet] 2009;43(3). [acesso em 10 ago 2012]. Disponível: http://dx.doi.org/10.1590/S008062342009000300015

20. Al-Aama T. Falls in the elderly: spectrum and prevention. Can Fam Physician. [Internet] 2011; 57 [acesso em 10 set 2012]. Disponível: http://www.cfp. ca/content/57/7/771.full.pdf

21. Vikhireva O, Pikhart $H$, Pajak A, Kubinova R, Malyutina $S$, Peasey $A$, et al. Non-fatal injuries in three Central and Eastern European urban population samples: the HAPIEE study. Eur. J. Public Health. [internet] 2009;20(6). [acesso em 10 ago 2012]. Disponível: http:// www.ncbi.nlm.nih.gov/pmc/articles/PMC2989029/

22. Yee WY, Cameron PA, Bailey MJ. Road traffic injuries in the elderly. Emerg Med J. [Internet] 2006;23. [acesso em 20 set 2012]. Disponível: http://www.ncbi.nlm.nih. gov/pubmed/16381081

23. Malik AM, Dal NA, Talpur KAH. Road traffic injuries and their outcome in the elderly patients 60 years and above. Does age make a difference? J. Trauma Treat. [internet] 2012;1(4) [acesso em 10 set 2012]. Disponível: http://www.omicsgroup.org/ journals/2167-1222/2167-1222-1-129.pdf

24. Minayo MCS. Violência contra idosos: relevância para um velho problema. Cad. Saúde Publica. [Internet] 2003;19(3). [acesso em 10 ago 2012]. Disponível: http:// dx.doi.org/10.1590/S0102-311X2003000300010

25. Mello S, Zamboni C, Fontana R, Rodrigues F. Violência contra idoso: um velho estigma. Cogitare enferm. [Internet] 2011;16(4) [acesso em 12 set 2012]. Disponível: http://ojs.c3sl.ufpr.br/ojs2/index.php/ cogitare/article/view/21973/17137

26. Yeo YYC, Lee SK, Lim CY, Quek LS, Ooi SBS. A review of elderly injuries seen in a Singapore emergency department. Singapore Med J. [Internet] 2009;50(3) [acesso em 10 ago 2012]. Disponível: http://www.ncbi. nlm.nih.gov/pubmed/19352571 